\title{
Multiorgan Immune-Related Adverse Events During Treatment With Atezolizumab
}

\author{
Ganessan Kichenadasse, MBBS, FRACP1,2; John O. Miners, PhD ${ }^{1}$; Arduino A. Mangoni, MBBS, FRACP, PhD ${ }^{1}$;
} Andrew Rowland, $\mathrm{PhD}^{1}$; Ashley M. Hopkins, $\mathrm{PhD}^{1}$; and Michael J. Sorich, $\mathrm{PhD}^{1}$

\begin{abstract}
Background: Immune-related adverse events (irAEs) are known to occur in patients with cancer who are treated with immune checkpoint inhibitors. However, limited literature exists on the incidence, time of onset, and risk factors for irAEs, particularly those affecting multiple organs, associated with anti-PD-L1 inhibitors. Methods: A post hoc pooled analysis was conducted using individual patient data from atezolizumab monotherapy arms of 4 non-small cell lung cancer clinical trials. Incidence, clinical patterns, outcomes, and risk factors were investigated of selected organ-specific and multiorgan irAEs during treatment using the anti-PD-L1 inhibitor atezolizumab. Results: From a total of 1,548 patients, 730 irAE episodes were reported in 424 patients (27\%). Skin irAEs were the most common (42\%), followed by laboratory abnormalities (27\%) and endocrine $(11.6 \%)$, neurologic $(7.6 \%)$, and pulmonary $(6.2 \%)$ irAEs. A total of 84 patients (5.4\%) had multiorgan irAEs, 70 had 2,13 had 3 , and 1 had 4 different organs affected. "Skin plus" or "laboratory plus" were the most common irAE multiorgan clusters. Patients with multiorgan irAEs were more likely to be white and have a good performance status, a lower baseline neutrophil-lymphocyte ratio, and a good or intermediate lung immune prognostic index score. Multiorgan irAEs were also associated with improved overall survival (hazard ratio, $0.47 ; 95 \% \mathrm{Cl}, 0.28-0.78 ; P<.0001)$ but not with progression-free survival (hazard ratio, $0.92 ; 95 \% \mathrm{Cl}, 0.62-1.35 ; P=.74$ ) compared with the cohort with no irAEs. Conclusions: Multiorgan irAEs occurred in $5.4 \%$ of patients treated with atezolizumab in non-small cell lung cancer trials. Future trials should consider routine reporting of data on multiorgan toxicities in addition to organ-specific toxicities.
\end{abstract}

J Natl Compr Canc Netw 2020;18(9):1191-1199 doi: $10.6004 /$ jnccn.2020.7567

${ }^{1}$ Department of Clinical Pharmacology, College of Medicine and Public Health, and ${ }^{2}$ Department of Medical Oncology, Flinders Centre for Innovation in Cancer, Flinders Medical Centre/Flinders University, Bedford Park, South Australia, Australia.

\section{Background}

Immune checkpoint inhibitors (ICIs) commonly target CTLA-4, PD-1, and PD-L1, which promote inhibitory signals on immune effector T cells against cancer cells. ${ }^{1}$ Although ICIs have improved outcomes in several cancers, their use is also associated with significant adverse events (AEs), including death. ${ }^{2}$

In contrast to other anticancer therapies, ICIs cause heterogeneous toxicities through nonspecific immune activation affecting tissues and organs. Although guidelines exist for the diagnosis and treatment of ICI-related toxicities, there is no international consensus on the terminology used for their definition, diagnostic criteria, grading, and causal attribution, and little evidence supporting their management. ${ }^{3-5}$ ESMO guidelines divide ICI-related AEs into infusion reactions and immune-related AEs (irAEs). ${ }^{4}$ Other guidelines have used AEs of special interest or immune-mediated AEs interchangeably with irAEs. ${ }^{6}$ Their pathophysiology is poorly understood, with several proposed mechanisms leading to organ damage. ${ }^{7,8}$

In addition, the reported incidence and time course of irAEs in clinical trials have varied based on the type of checkpoint being targeted. A recent network meta-analysis identified that atezolizumab, an anti-PD-L1 inhibitor, had the best safety profile. ${ }^{9}$ Other analyses reported an incidence of up to $30 \%$ with PD-1/PD-L1 inhibitors, ${ }^{10} 72 \%$ with ipilimumab, ${ }^{11}$ and $88 \%$ with combined therapies. ${ }^{12}$

Although more than 1 autoimmune disease can occur in the same patient (polyautoimmunity), ${ }^{13}$ it is unclear whether multiple-organ irAEs have occurred in the same patient with cancer, and when they occur, whether they have occurred simultaneously or serially. Moreover, the reported irAEs in clinical trials are often limited to single organs, preventing accurate information regarding the incidence and time course of multiple irAEs. ${ }^{14}$ Such an occurrence of multiple irAEs in the same patient may influence their treatment decisions for both the AEs and the cancer. There are additional

See page 1287 for related commentary. 
complexities, such as the use of polypharmacy and the involvement of various specialties for the management of AEs. Hence, it is important to have a thorough understanding of multiorgan irAEs from ICIs. Using pooled data from 4 prospective clinical trials in patients with non-small cell lung cancer treated using atezolizumab monotherapy, we analyzed the incidence, severity, time course, treatment, outcomes, and risk factors of various organ-specific irAEs and their relationships with response rates/survival outcomes.

\section{Methods}

\section{Study Population}

Individual patient data from the OAK (ClinicalTrials.gov identifier: NCT02008227), ${ }^{15}$ POPLAR (NCT01903993), ${ }^{16}$ BIRCH (NCT02031458), ${ }^{17}$ and FIR (NCT01846416) ${ }^{18}$ clinical trials were analyzed via established protocols for clinical data-sharing. ${ }^{19}$ This secondary analysis of trial data was exempted from review by the local ethics committee. Only atezolizumab-treated cohorts were included in the analysis.

\section{Definition of AEs}

AEs for which the investigator attributed causality to atezolizumab and that occurred while patients were on atezolizumab or within 30 days after the last dose were adjudicated as treatment-related AEs (trAEs). irAEs were defined as per prespecified study protocols (ie, AEs of special interest as conditions suggestive of an autoimmune disorder resulting from atezolizumab use). These events were organ-specific and included endocrine, ocular, gastrointestinal, hepatobiliary and pancreatic, neurologic, pulmonary, rheumatologic, skin, laboratory abnormalities, and other miscellaneous organ-specific irAEs. Definition terms for each organ-specific irAE are summarized in Table 1 . The single-organ irAE cohort was defined as patients with 1 organ system affected, and the multiorgan irAE cohort was defined as patients with $>1$ organ system involved. Concurrent multiorgan irAE was defined as the onset of irAEs within 7 days of each other, and sequential multiorgan irAEs as onset $>7$ days between each. An irAE "episode" was recorded at the time of reporting and when the grade (ie, the severity) increased, decreased, or resolved. An irAE that had not resolved or changed grade was counted as a single episode. AEs were graded using NCI CTCAE version 4.0, with grades 3-5 considered severe irAEs.

\section{Outcomes}

Primary objectives were the incidence, grades, and time course of irAEs resulting from atezolizumab. Secondary objectives were the incidence and grades of trAEs, progression-free survival (PFS), and overall survival (OS). PFS was assessed using RECIST (version 1.1 ) or a modified RECIST. Exploratory analyses investigated risk factors for irAEs and the relationship between irAEs and survival outcomes.

Baseline age, sex, race, tumor characteristics, serum lactate dehydrogenase and C-reactive protein (CRP) levels, lung immune prognostic index (LIPI), neutrophil-tolymphocyte ratio, and various subpopulations of white cells were evaluated for associations with incidence of irAEs.

\section{Statistical Analysiss}

Two-sided statistical tests were conducted using R version 3.4.3 (R Foundation for Statistical Computing), and $P<.05$ was considered statistically significant. The Fisher exact test for categorical data and the Wilcoxon test for continuous data were used for the association between baseline characteristics and irAEs. Timing of onset of

Table 1. Medical Dictionary for Regulatory Activities Terms Used to Identify Organ-Specific irAEs

\begin{tabular}{|c|c|}
\hline Organ-Specific irAE & Reported Terms \\
\hline Skin & $\begin{array}{l}\text { Rash, maculopapular rash, macular rash, popular rash, erythematous rash, dermatitis, psoriasis, eczema, } \\
\text { lichen planus, generalized rash, pruritic rash, vitiligo, dermatomyositis, or pemphigoid }\end{array}$ \\
\hline Endocrine & $\begin{array}{l}\text { Hypothyroidism, hyperthyroidism, adrenal insufficiency, thyroiditis, hypophysitis, adrenal insufficiency, } \\
\text { type } 1 \text { diabetes mellitus, and increased or decreased thyroid stimulating hormone }\end{array}$ \\
\hline Gastrointestinal & Colitis or duodenitis \\
\hline Neurologic & Peripheral neuropathy, polyneuropathy, or Guillain-Barré syndrome \\
\hline Pulmonary & Pneumonitis, interstitial pneumonitis, or organizing pneumonia \\
\hline Hepatobiliary and pancreatic & Cholangitis, hepatitis, or pancreatitis \\
\hline Ocular & Episcleritis, uveitis, optic neuritis, or endocrine ophthalmopathy \\
\hline Rheumatologic & Rheumatoid arthritis, polyarthritis, rheumatic disorder, or autoimmune arthritis \\
\hline Laboratory irAEs & Elevated serum liver enzymes or bilirubin, lipase, or amylase level \\
\hline Miscellaneous & Those which did not fit under the above organs, such as cardiovascular system, kidneys, and pericardium \\
\hline
\end{tabular}

Abbreviation: irAE, immune-related adverse event. 
irAEs was visually displayed using a Swimmer plot. Logistic regression analysis was performed to investigate the association between irAEs and best overall response. Simple Cox and time-dependent Cox proportional hazards regression were used to model the association between the irAE cohorts and survival outcomes (OS and PFS) and reported as hazard ratios (HRs) with 95\% confidence intervals. Survival curves were estimated using Kaplan-Meier analysis.

\section{Results}

Overall Incidence and Severity of AEs

The analysis included 1,548 patients who received at least 1 dose of atezolizumab. One or more trAEs occurred in 1,000 patients (65\%), with grade $3-5$ trAEs in $13 \%$ of patients across the trials. A total of $730 \mathrm{irAE}$ episodes were reported across 424 patients (27\%) who had $\geq 1$ irAEs. Median time of onset was 49 days (interquartile range, 21-130 days).

\section{Baseline Characteristics of Patients Who Developed irAEs}

Tables 2 and 3 present the characteristics of patients with irAEs. Twelve percent of irAEs occurred within the first 42 days of atezolizumab use. Patients with irAEs were more likely to be Asian and have good performance status, lower CRP levels, differential changes in subpopulations of white cells, a good LIPI score, and a lower neutrophilto-lymphocyte ratio score. Details of organ-specific irAEs are described in Table 4. Figure 1 shows selected singleorgan irAEs and their time of onset. Skin irAEs were the most common single-organ irAE, followed by laboratory abnormalities and other organs. Notably, there were no reported myocarditis or severe cutaneous reactions, such as bullous pemphigoid. The investigators did not consider anemia (36 episodes), thrombocytopenia (7 episodes), neutropenia (5 episodes), and lymphopenia (3 episodes) to be irAEs.

\section{Treatment of irAEs and Their Outcomes}

Overall, $7.5 \%$ of the 730 episodes of irAEs resulted in hospitalization, which was required in almost half of grade 3 or 4 events and in $5.7 \%$ of grade 1 or 2 events. A total of 109 (15\%) irAE episodes were treated with systemic corticosteroids, of which 31 episodes $(30 \%)$ were grade 1 or 2 and the rest were grade $\geq 3$, including 1 grade 5 event (1 death). Atezolizumab was interrupted in $14 \%(102 / 730)$ of all episodes and was permanently discontinued in $2.5 \%(18 / 730)$. At the time of last follow-up, most irAE episodes $(\mathrm{n}=438 ; 60 \%)$ were resolved and $28 \%(n=204)$ had ongoing irAEs. Nine episodes resulted in sequelae despite resolution of the irAE, and the remainder $(11 \%)$ were resolving at last follow-up. Only 1 death from irAEs (pneumonitis) was reported.
Second-Line Treatment for irAEs

Second-line immunosuppressants for the treatment of irAEs, after systemic corticosteroid failure, was required in 5 patients. One patient received infliximab for colitis and experienced complete resolution, 1 required oral mycophenolate mofetil for hepatitis, 1 received anakinra for arthritis, and 2 had topical cyclosporine A for ocular irAEs. Atezolizumab was discontinued permanently in the patient with hepatitis. All other patients requiring second-line immunosuppressants received further atezolizumab.

\section{Multiorgan irAEs}

Of the 424 patients with irAEs, 340 had 1 organ-specific irAE (see Tables 2 and 3$)$. The remainder $(n=84 ; 5.4 \%$ $[84 / 1,548]$ of all atezolizumab-treated patients and $19.8 \%$ [84/424] of all patients with irAEs) had multiorgan irAEs. Among those who had multiorgan irAEs, 70 patients (83.3\%) had 2 organ systems affected by irAEs, 13 (15.5\%) had 3, and 1 had 4 (Figure 2); 12 had concurrent-onset irAEs and the remainder $(\mathrm{n}=72)$ had sequential onset. Those who had multiorgan irAEs were more likely to be white and have an ECOG performance status of 0 and a lower baseline CRP level and neutrophil-lymphocyte ratio than those who developed single-organ irAEs.

The combinations of organs affected by irAEs were "skin irAE plus," "laboratory irAE plus without skin," or "other combinations without skin or laboratory irAE." A total of 60 patients were in the skin irAE plus cluster, 9 were in the laboratory irAE plus without skin cluster, and the remaining 15 had other combinations.

\section{Multiorgan irAEs and Cancer Outcomes}

The development of multiorgan irAEs was associated with improved response rates, PFS, and OS. The best overall response (combined complete and partial response) was significantly higher among those who developed multiorgan irAE than those with single-organ irAEs or without irAEs ( $42 \%, 23 \%$, and $12 \%$, respectively), with an odds ratio of 2.18 (95\% CI, 1.5-2.9) for singleorgan irAEs (vs no irAEs) and 5.32 (95\% CI, 3.3-8.5) for multiorgan irAEs (vs no irAEs) $(P<.0001)$. Median PFS using a simple Cox model was significantly improved with multiorgan irAEs (7.2 months; $95 \%$ CI, 5.4-12.4) vs single-organ irAEs (4.2 months; 95\% CI, 3.9-5.5) and no irAEs (2.7 months; 95\% CI, 2.5-2.8; $P \leq .001$ ) (Figure 3A). Similarly, median OS measured with a simple Cox model was significantly improved in patients with multiorgan irAEs (not reached; 95\% CI, 23.5-not reached) versus those with single-organ irAEs (20.1 months; $95 \% \mathrm{CI}$, 16.0-23.3) and no irAEs (10.3 months; 95\% CI, 9.5-11.4; $P<.001$ ) (Figure 3B). HRs calculated using a time-dependent Cox regression model for OS was 0.47 (95\% CI, 0.28-0.78) for multiorgan irAEs (vs no irAEs) and 0.69 (95\% CI, 0.57-0.85) 
Table 2. Baseline Clinical Characteristics and Outcomes of Atezolizumab-Treated Patients

\begin{tabular}{|c|c|c|c|c|c|}
\hline & $\begin{array}{l}\text { Patients } \\
\text { n (\%) }\end{array}$ & $\begin{array}{l}\text { irAEs } \\
\text { n (\%) }\end{array}$ & $\begin{array}{c}\text { Single-Organ irAEs } \\
\mathbf{n}(\%)\end{array}$ & $\begin{array}{c}\text { Multiorgan irAEs } \\
\text { n (\%) }\end{array}$ & $P$ Value ${ }^{a}$ \\
\hline Total, N & 1,548 & 1,124 & 340 & 84 & \\
\hline $\mathrm{BIRCH}^{17}$ & $659(43 \%)$ & $487(43 \%)$ & $138(41 \%)$ & $34(40 \%)$ & \\
\hline $\mathrm{FIR}^{18}$ & $138(9 \%)$ & 108 (10\%) & $24(7 \%)$ & $6(7 \%)$ & \\
\hline Age, y & $64(57-70)$ & $63(56-70)$ & $66(58-72)$ & $62(57-68)$ & .14 \\
\hline Sex & & & & & .75 \\
\hline Male & $936(60 \%)$ & $673(60 \%)$ & 211 (62\%) & 52 (62\%) & \\
\hline Female & $612(40 \%)$ & $451(40 \%)$ & $129(38 \%)$ & $32(38 \%)$ & \\
\hline Other & $66(4 \%)$ & $48(4 \%)$ & $17(5 \%)$ & $1(1 \%)$ & \\
\hline Missing & $35(2 \%)$ & $26(2 \%)$ & $8(2 \%)$ & $1(1 \%)$ & \\
\hline ECOG performance status & & & & & .031 \\
\hline 0 & 535 (35\%) & 366 (33\%) & 131 (39\%) & $38(45 \%)$ & \\
\hline 1 & $1,002(65 \%)$ & 749 (67\%) & $208(61 \%)$ & 45 (54\%) & \\
\hline 2 & $8(1 \%)$ & $6(1 \%)$ & $1(<1 \%)$ & $1(1 \%)$ & \\
\hline Missing & $3(<1 \%)$ & $3(<1 \%)$ & $0(0 \%)$ & $0(0 \%)$ & \\
\hline Histology & & & & & .20 \\
\hline Nonsquamous & $1,155(72 \%)$ & 797 (71\%) & $258(76 \%)$ & $60(71 \%)$ & \\
\hline Never & 270 (17\%) & 201 (18\%) & $61(18 \%)$ & $8(10 \%)$ & \\
\hline Previous & $1,081(70 \%)$ & 777 (69\%) & 245 (72\%) & 59 (70\%) & \\
\hline Current & 197 (13\%) & $146(13 \%)$ & 34 (10\%) & 17 (20\%) & \\
\hline Missing & $1(0.1 \%)$ & $1(0.1 \%)$ & $0(0 \%)$ & $0(0 \%)$ & \\
\hline
\end{tabular}

Abbreviation: irAE, immune-related adverse event.

a $P$ values per Fisher exact test for categorical data and Wilcoxon test for continuous data.

bNumber of prior treatments in the locally advanced or metastatic setting.

for single-organ irAEs (vs no irAEs) $(P<.0001)$. However, the occurrence of multiorgan irAEs was not associated with an improved PFS in the time-dependent Cox model (HR, 0.92; 95\% CI, 0.62-1.35), nor was the occurrence of single-organ irAEs (HR, 0.95; 95\% CI, $0.81-1.11 ; P=.74)$.

\section{Discussion}

We comprehensively assessed multiorgan irAEs associated with ICIs using a large dataset from clinical trials. One-fifth of patients treated using atezolizumab developed at least 1 irAE, whereas $5.4 \%$ had multiorgan irAEs. Mortality from irAEs and irAEs leading to permanent discontinuation of atezolizumab was uncommon, with most patients recovering without sequelae. Any irAEs, including multiorgan irAEs, were significantly associated with better tumor response and survival outcomes.

Although the occurrence of polyautoimmunity is well-known in the rheumatologic literature, there has been little information on the occurrence of multisystem 


\section{Table 3. Baseline Laboratory Characteristics of Atezolizumab-Treated Patients}

\begin{tabular}{|c|c|c|c|c|c|}
\hline & $\begin{array}{l}\text { Patients } \\
\text { n (\%) }\end{array}$ & $\begin{array}{l}\text { No irAEs } \\
n(\%)\end{array}$ & $\begin{array}{c}\text { Single-Organ irAEs } \\
\mathbf{n}(\%)\end{array}$ & $\begin{array}{c}\text { Multiorgan irAEs } \\
\mathbf{n}(\%)\end{array}$ & $P$ Value $^{\mathrm{a}}$ \\
\hline Total, N & 1,548 & 1,124 & 340 & 84 & \\
\hline PD-L1 expression & & & & & .69 \\
\hline Negative & 528 (34\%) & 383 (34\%) & 119 (35\%) & $26(31 \%)$ & \\
\hline Positive & $1,015(65 \%)$ & 737 (65\%) & $220(64 \%)$ & $58(69 \%)$ & \\
\hline Missing & $5(<1 \%)$ & $4(<1 \%)$ & $1(<1 \%)$ & $0(0 \%)$ & \\
\hline$\leq \mathrm{ULN}$ & $913(59 \%)$ & $649(58 \%)$ & $211(62 \%)$ & $53(63 \%)$ & \\
\hline$>$ ULN & $584(38 \%)$ & 439 (39\%) & $116(34 \%)$ & 29 (35\%) & \\
\hline Missing & $51(3 \%)$ & $36(3 \%)$ & $13(4 \%)$ & $2(2 \%)$ & \\
\hline Neutrophil count ( & & & & & .002 \\
\hline Median (IQR) & $5(3.7-6.7)$ & $5.1(3.8-7.0)$ & $4.7(3.7-6.0)$ & $4.9(3.5-6.3)$ & \\
\hline Missing & $90(5.8 \%)$ & $67(6 \%)$ & $18(5.3 \%)$ & $5(6 \%)$ & \\
\hline Basophil count (10 & & & & & .14 \\
\hline Median (IQR) & $0.02(0-0.05)$ & $0.02(0-0.05)$ & $0.03(0-0.06)$ & $0.03(0-0.06)$ & \\
\hline Missing & $124(8 \%)$ & $93(8.2 \%)$ & $26(7.6 \%)$ & $5(5.9 \%)$ & \\
\hline Eosinophil count & & & & & .12 \\
\hline Median (IQR) & $0.14(0.09-0.24)$ & $0.14(0.09-0.23)$ & $0.15(0.10-0.29)$ & $0.15(0.10-0.30)$ & \\
\hline Missing & $108(6.9 \%)$ & $79(7 \%)$ & $24(7 \%)$ & $5(5.9 \%)$ & \\
\hline Monocyte count & & & & & .28 \\
\hline Median (IQR) & $0.61(0.48-0.84)$ & $0.63(0.48-0.85)$ & $0.60(0.48-0.80)$ & $0.60(0.48-0.80)$ & \\
\hline Missing & $96(6.2 \%)$ & $72(6.4 \%)$ & $19(5.6 \%)$ & $5(5.9 \%)$ & \\
\hline CD8 count $\left(10^{\circ} / \mathrm{L}\right)$ & & & & & .78 \\
\hline Median (IQR) & $293(177-455)$ & $291(175-450)$ & $292(184-446)$ & $313(218-468)$ & \\
\hline Missing & $75(5 \%)$ & $56(5 \%)$ & $15(4 \%)$ & $4(5 \%)$ & \\
\hline Neutrophil-to-lymp & & & & & .007 \\
\hline Median (IQR) & $2.2(1.6-3.2)$ & $2.3(1.7-3.3)$ & $2.1(1.6-2.8)$ & $2.0(1.4-2.8)$ & \\
\hline Missing & $29(1.9 \%)$ & $23(2 \%)$ & $6(1.8 \%)$ & $0(0 \%)$ & \\
\hline C-reactive protein & & & & & $<.001$ \\
\hline Median (IQR) & $14(4-42)$ & 15 (4-48) & $13(5-45)$ & $11(4-32)$ & \\
\hline Missing & $43(3 \%)$ & $26(4 \%)$ & $9(2 \%)$ & $8(3 \%)$ & \\
\hline Lung immune pros & & & & & .010 \\
\hline 0 & $678(44 \%)$ & $464(41 \%)$ & $172(51 \%)$ & 42 (50\%) & \\
\hline 1 & $631(41 \%)$ & 472 (42\%) & $125(37 \%)$ & 34 (40\%) & \\
\hline 2 & $180(12 \%)$ & 145 (13\%) & $29(9 \%)$ & $6(7 \%)$ & \\
\hline Missing & $59(4 \%)$ & $43(4 \%)$ & $14(4 \%)$ & $2(2 \%)$ & \\
\hline
\end{tabular}

Abbreviations: IQR, interquartile ratio; irAE, immune-related adverse event; ULN, upper limit of normal.

${ }^{a} P$ values per Fisher exact test for categorical data and Wilcoxon test for continuous data. 
Table 4. Details of Organ-Specific irAEs

\begin{tabular}{|c|c|c|c|c|}
\hline \multirow[b]{2}{*}{ Organ-Specific irAE } & \multirow{2}{*}{$\begin{array}{l}\text { Unique Episodes } \\
\text { of irAEs, } n\end{array}$} & \multirow[b]{2}{*}{ Type of irAE (\%) } & \multicolumn{2}{|c|}{ Grade (\%) } \\
\hline & & & $1 / 2$ & $\geq 3$ \\
\hline Skin & 306 & $\begin{array}{l}\text { Skin rash }(89 \%) \text {, eczema }(4.6 \%) \text {, psoriasis }(2.3 \%) \text {, lichen } \\
\text { planus }(1 \%) \text {, dermatomyositis, vitiligo, and bullous } \\
\text { pemphigoid ( }<1 \% \text { each) }\end{array}$ & 86 & 14 \\
\hline Laboratory & 197 & $\begin{array}{l}\text { Liver enzyme abnormalities were the majority followed by } \\
\text { increased bilirubin, lipase, or amylase }\end{array}$ & 80 & 20 \\
\hline Endocrine & 85 & $\begin{array}{l}\text { Thyroid gland-related (hyperthyroidism or } \\
\text { hypothyroidism, } 92.5 \%) \text {, adrenal gland-related ( } 4.7 \%) \text {, } \\
\text { type } 1 \text { diabetes mellitus ( } 2.4 \%) \text {, hypophysitis (1.2\%) }\end{array}$ & 95.3 & 4.7 \\
\hline Neurologic & 56 & $\begin{array}{l}\text { Peripheral neuropathy (84\%), polyneuropathy }(9 \%), \\
\text { Guillain-Barré syndrome (7\%) }\end{array}$ & 66 & 7 \\
\hline Pulmonary & 45 & Pneumonitis (95\%) and organizing pneumonia (5\%) & 57.8 & $42.2^{\mathrm{a}}$ \\
\hline Gastrointestinal & 15 & Colitis (93.3\%) and duodenitis (6.7\%) & 66.7 & 33.3 \\
\hline Ocular & 9 & $\begin{array}{l}\text { Uveitis and optic neuritis ( } 33.3 \% \text { each), endocrine } \\
\text { ophthalmopathy }(22.2 \%) \text {, episcleritis }(11.1 \%)\end{array}$ & 88.8 & 11.2 \\
\hline Rheumatologic & 8 & $\begin{array}{l}\text { Rheumatoid arthritis and polymyalgia rheumatica ( } 37.5 \% \\
\text { each), polyarthritis and rheumatic disorder ( } 12.5 \% \text { each) }\end{array}$ & 100 & 0 \\
\hline Miscellaneous & 5 & $\begin{array}{l}\text { Pericarditis ( } 40 \%) \text { and cytokine release syndrome, Henoch- } \\
\text { Schönlein purpuric nephritis, and vasculitis ( } 20 \% \text { each) }\end{array}$ & 60 & 40 \\
\hline Hepatobiliary and pancreatic & 4 & Pancreatitis (75\%) and cholangitis (25\%) & 100 & 0 \\
\hline
\end{tabular}

Abbreviation: irAE, immune-related adverse event. ancludes 1 death.

irAEs until recently, likely because of underrecognition and lack of routine reporting. Shankar et $\mathrm{al}^{20}$ recently reported an incidence of $5 \%$ for multisystem irAEs among 319 patients with lung cancer treated using anti-PD-1/PD-L1 therapies, similar to our findings. However, in contrast to our study, pneumonitis was the dominant irAE, with pneumonitis/dermatitis as the most common multiorgan irAEs. In the current study, skin plus or laboratory plus clusters were the most dominant. Furthermore, our study included patients with lung cancer treated with a single drug (atezolizumab) in clinical trials, in contrast to the Shankar et $\mathrm{al}^{20}$ study in which several types of ICIs were given to patients in a real-world setting.

The differences in the incidence of organ-specific irAEs between studies may be related to the lack of uniform definitions of organ-specific irAEs across trials. Maughan et al ${ }^{14}$ previously identified important differences in the definition of irAEs between atezolizumab, nivolumab, and pembrolizumab trials and recommended standardized definitions. For the current analysis, irAEs were defined according to trial protocols as autoimmune conditions based on known mechanisms of ICIs.

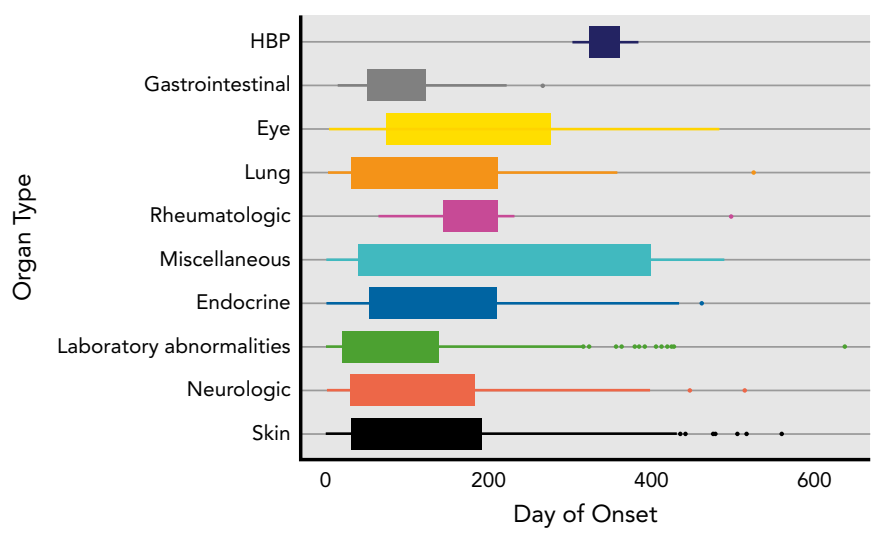

Figure 1. Time of onset of organ-specific immune-related adverse events.

Abbreviation: HBP, hepatobiliary and pancreatic. 


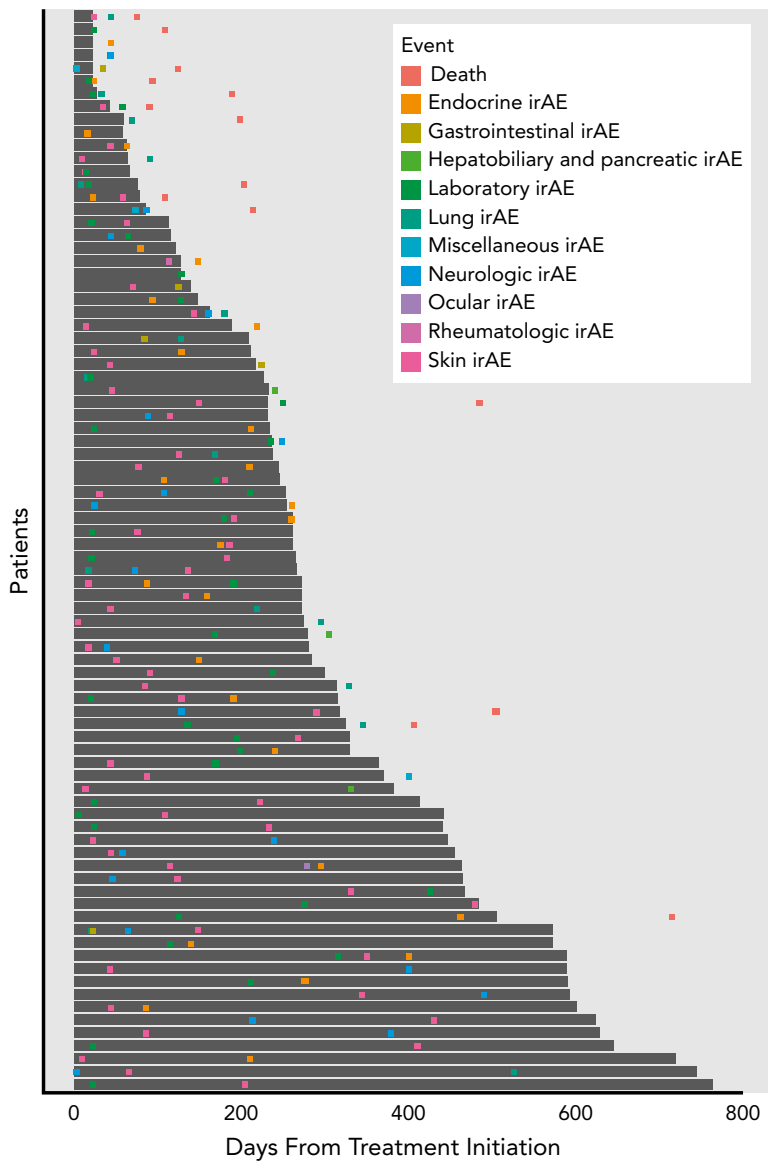

Figure 2. Swimmer plot showing time of onset of multiple irAEs and deaths from all causes in 84 patients. Each bar indicates duration of atezolizumab treatment.

Abbreviation: irAE, immune-related adverse event.

The type of single-organ irAE in our study was dominated by cutaneous irAEs, followed by laboratory abnormalities and endocrine irAEs. This contrasts with a previous analysis of anti-PD-1/PD-L1 drugs, in which any grade of endocrine irAE was the most common organ system affected, followed by pneumonitis. ${ }^{21}$ Of interest, neurologic irAEs, especially neuropathy, emerged as important irAEs in our analysis. The reason for the relatively high incidence of neuropathy as an irAE from atezolizumab is unknown. It is also unclear whether the incidence of neurologic irAEs is similar with other ICIs or whether they were underrecognized or underreported in previous trials. Future studies should consider a prospective evaluation of neuropathy during treatment.

Among the baseline characteristics associated with the development of irAEs, Asian race and a good LIPI score were statistically significant. Prior studies have similarly reported associations with Asian race ${ }^{22}$; however, the association with a good LIPI score has not been previously reported.
Prior studies have suggested a relationship between the occurrence of irAEs and tumor response and survival with ICIs for various cancers. ${ }^{23,24}$ Presence of a generalized immunogenic state during ICI treatment may contribute toward better cancer control along with damage to normal organs that manifests as irAEs. We identified a similar association between the occurrence of irAEs and best overall response, PFS, and OS. Moreover, the occurrence of multiorgan irAEs was strongly associated with improved OS but not PFS, with time-dependent Cox models indicating a possible immortal time bias, confounding the results in the simple Cox model.

In addition, the definition of abnormal blood results and their attribution to organ-specific damage needs further consideration. Whether laboratory abnormalities alone constitute irAEs requires international consensus. None of the available guidelines provide definitions for organ-specific irAEs. We believe that our understanding of the pathophysiology, incidence, and management of irAEs can only be improved by using uniform definitions for the development of evidence-based guidance.

Although the diagnosis and characterization of irAEs remain inconsistent and challenging, ${ }^{25}$ the expert consensus-based Trial Reporting of Immuno-Oncology (TRIO) recommendations are likely to improve the reporting of ICIs in clinical trials. However, the TRIO guidance does not recognize or mandate reporting on multiorgan irAEs. ${ }^{25}$ As described in our analysis, multiorgan toxicities occur in a significant proportion of patients treated with ICIs, and these may have associations with treatment response and outcomes. Hence, their recognition and reporting should become part of published manuscripts and the routine care of patients undergoing with ICI treatment.

Polyautoimmunity phenotypes often occur because of common genetic factors, immune pathogenic mechanisms, and an established set of risk factors, such as female predominance, family history, smoking history, and Native American race; it is unclear whether multiorgan irAEs have any risk factors. ${ }^{26}$ In the current study, white race and good ECOG performance status were associated with multiorgan irAEs. In contrast to the polyautoimmunity phenotype, sex and smoking history were not associated with multiorgan irAEs. Further studies should evaluate any underlying genetic risk factors that may predispose certain individuals who receive ICIs to multiorgan irAEs, using data through international collaboration. ${ }^{27}$

Prior reports on polyautoimmunity also identified that the treatment of multiple conditions adds complexity to their care. ${ }^{13,26,28}$ Similarly, treatment of patients with multiorgan irAEs poses other challenges, such as need for endocrine replacement therapies, use of immunosuppressants at the same time, possible need for second-line immunosuppressants, and secondary 
A

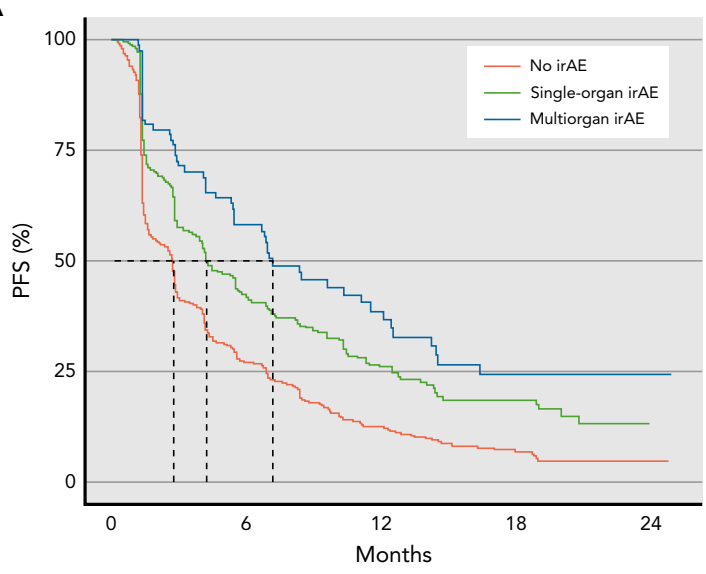

B

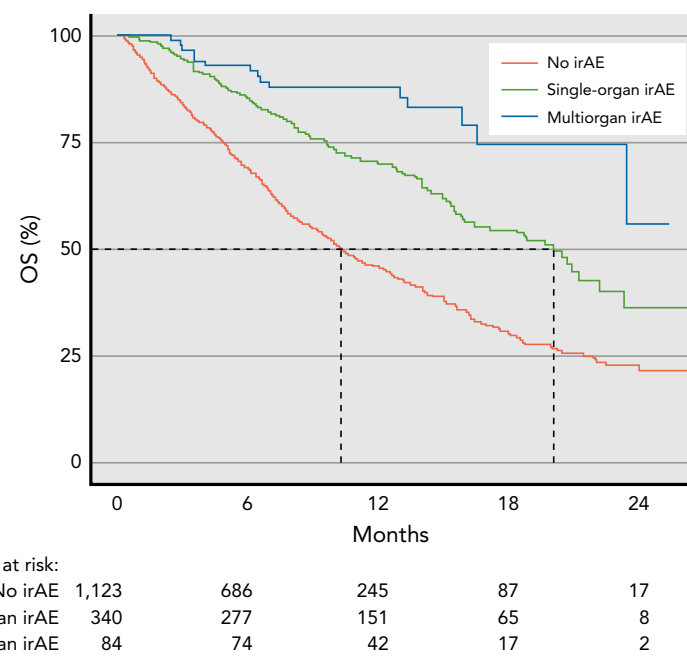

Figure 3. Simple Cox model showing association between irAEs and (A) PFS and (B) OS outcomes. Abbreviations: irAE, immune-related adverse event; OS, overall survival; PFS, progression-free survival.

complications, such as high blood glucose levels and infections from corticosteroids. In the current study, because of the limited number of patients, it was unclear whether those with multiorgan irAEs had an increased need for second-line immunosuppressants.

\section{Strengths}

This study highlights a previously underrecognized issue of multiorgan irAEs arising from ICIs. This is the largest analysis of uniformly treated patients with non-small cell lung cancer who developed multiorgan irAEs from an anti-PD-L1 inhibitor. Data were collected prospectively through multicenter international clinical trials, thereby improving the accuracy of the information. Moreover, the association between the occurrence of irAEs and survival outcomes was evaluated using time-dependent Cox models to avoid bias from different treatment durations. ${ }^{29}$

\section{Limitations}

Our study has several limitations. First, the analysis is a post hoc exploratory analysis and therefore should be considered hypothesis-generating and confirmed in other studies. Second, the data were from lung cancer trials of atezolizumab; it is unclear whether a similar pattern of multiorgan irAEs occurs with other cancer types or ICIs. Third, the data had investigator-reported AEs and there was no external validation/confirmation of AEs. Finally, the irAEs analyzed were organ-specific, and did not include constitutional symptoms such as fatigue and fever, which could be related to cytokines released by activated immune cells. These toxicities are not traditionally regarded as irAEs, although they may have immune etiology. Despite these study limitations, we have shown that multiorgan irAEs need appropriate recognition and evaluation.

\section{Conclusions}

Multiorgan irAEs were reported in $5.4 \%$ of patients treated with atezolizumab in lung cancer trials. Health professionals involved in the care of patients undergoing ICI therapies should recognize that irAEs can manifest as multiple organ system damage. Future trial reporting should consider the incorporation of data on multiorgan toxicities in addition to single-organ-specific toxicities.

Submitted January 25, 2020; accepted for publication March 27, 2020.

Author contributions: Study concept and design: Kichenadasse, Hopkins, Sorich. Funding: Hopkins, Sorich. Data acquisition: Hopkins, Sorich. Data analysis and interpretation: Miners, Mangoni, Rowland, Hopkins, Sorich Statistical analysis: Kichenadasse. Technical support: Miners, Rowland, Hopkins. Technical/Material support: Mangoni. Supervision: Miners, Mangoni, Rowland, Hopkins, Sorich. Manuscript preparation: All authors. Critical revision for intellectual content: All authors.

Disclosures: Dr. Rowland has disclosed that he has received grant/research support from Pfizer. Dr. Sorich has disclosed that he has received grant/research support from Pfizer. The remaining authors have disclosed that they have not received any financial consideration from any person or organization to support the preparation, analysis, results, or discussion of this article.

Funding: This work was supported by a research grant by Cancer Council South Australia (1159924 and 1127220 to Dr. Sorich) and the National Breast Cancer Foundation (grant PF-17-007 to Dr. Hopkins)

Disclaimer: Individual-participant data used were accessed via https:// www.clinicalstudydatarequest.com, according to Roche's policy and process for clinical study data-sharing.

Correspondence: Ganessan Kichenadasse, MBBS, FRACP, Department of Medical Oncology, Flinders Centre for Innovation in Cancer, Flinders Medica Centre/Flinders University, Bedford Park, South Australia 5042, Australia. Email: ganessan.kichenadasse@flinders.edu.au 


\section{References}

1. Wei SC, Duffy CR, Allison JP. Fundamental mechanisms of immune checkpoint blockade therapy. Cancer Discov 2018;8:1069-1086.

2. Wang DY, Salem JE, Cohen JV, et al. Fatal toxic effects associated with immune checkpoint inhibitors: a systematic review and meta-analysis. JAMA Oncol 2018;4:1721-1728.

3. Brahmer JR, Lacchetti C, Thompson JA. Management of immune-related adverse events in patients treated with immune checkpoint inhibitor therapy: American Society of Clinical Oncology clinical practice guideline summary. J Oncol Pract 2018;14:247-249.

4. Haanen J, Carbonnel F, Robert C, et al. Management of toxicities from immunotherapy: ESMO clinical practice guidelines for diagnosis, treatment and follow-up. Ann Oncol 2017;28(Suppl 4):iv119-142.

5. Puzanov I, Diab A, Abdallah K, et al. Managing toxicities associated with immune checkpoint inhibitors: consensus recommendations from the Society for Immunotherapy of Cancer (SITC) Toxicity Management Working Group. J Immunother Cancer 2017;5:95.

6. Wang PF, Chen Y, Song SY, et al. Immune-related adverse events associated with anti-PD-1/PD-L1 treatment for malignancies: a meta-analysis. Front Pharmacol 2017;8:730.

7. Postow MA, Sidlow R, Hellmann MD. Immune-related adverse events associated with immune checkpoint blockade. N Engl J Med 2018;378: 158-168.

8. Hirschhorn D, Ricca J, Gasmi B, et al. A delicate interplay between adaptive and innate immunity caused by immunotherapy triggers tumor immunity and aseptic inflammation. J Immunol 2018;200 (1 Suppl):178.42

9. Xu C, Chen YP, Du XJ, et al. Comparative safety of immune checkpoint inhibitors in cancer: systematic review and network meta-analysis. BMJ 2018;363:k4226.

10. Nishijima TF, Shachar SS, Nyrop KA, et al. Safety and tolerability of PD-1/ PD-L1 inhibitors compared with chemotherapy in patients with advanced cancer: a meta-analysis. Oncologist 2017;22:470-479.

11. Bertrand A, Kostine M, Barnetche T, et al. Immune related adverse events associated with anti-CTLA-4 antibodies: systematic review and metaanalysis. BMC Med 2015;13:211.

12. Gu L, Khadaroo PA, Su H, et al. The safety and tolerability of combined immune checkpoint inhibitors (anti-PD-1/PD-L1 plus anti-CTLA-4): a systematic review and meta-analysis. BMC Cancer 2019;19:559.

13. Matusiewicz A, Stróżyńska-Byrska J, Olesińska M. Polyautoimmunity in rheumatological conditions. Int J Rheum Dis 2019;22:386-391.

14. Maughan BL, Bailey E, Gill DM, et al. Incidence of immune-related adverse events with program death receptor-1- and program death receptor-1 ligand-directed therapies in genitourinary cancers. Front Oncol $2017 ; 7: 56$.

15. Rittmeyer A, Barlesi F, Waterkamp D, et al. Atezolizumab versus docetaxel in patients with previously treated non-small-cell lung cancer (OAK): a phase 3, open-label, multicentre randomised controlled trial. Lancet 2017:389:255-265.
16. Fehrenbacher $L$, Spira A, Ballinger $M$, et al. Atezolizumab versus docetaxe for patients with previously treated non-small-cell lung cancer (POPLAR): multicentre, open-label, phase 2 randomised controlled trial. Lancet 2016;387:1837-1846.

17. Peters S, Gettinger S, Johnson ML, et al. Phase II trial of atezolizumab as first-line or subsequent therapy for patients with programmed deathligand 1-selected advanced non-small-cell lung cancer (BIRCH). J Clin Oncol 2017;35:2781-2789.

18. Spigel DR, Chaft JE, Gettinger S, et al. FIR: efficacy, safety, and biomarker analysis of a phase II open-label study of atezolizumab in PD-L1-selected patients with NSCLC. J Thorac Oncol 2018;13:1733-1742.

19. Clinical Study Data Request. Sponsor specific details: Roche. Accessed March 31, 2020. Available at: https://www.clinicalstudydatarequest.com/ Study-Sponsors/Study-Sponsors-Roche.aspx

20. Shankar B, Zhang J, Barasa D, et al. Multisystem immune-related adverse events from anti-PD-1/PD-L1 in patients with lung cancer: incidence, clinical patterns, management, and outcomes [abstract]. J Clin Oncol 2019;37(Suppl):Abstract 84.

21. Wang Y, Zhou S, Yang F, et al. Treatment-related adverse events of PD-1 and PD-L1 inhibitors in clinical trials: a systematic review and metaanalysis. JAMA Oncol 2019;5:1008-1019.

22. Peng L, Wu YL. Immunotherapy in the Asiatic population: any differences from Caucasian population? J Thorac Dis 2018;10(Suppl 13):S1482-1493.

23. Petrelli F, Grizzi G, Ghidini M, et al. Immune-related adverse events and survival in solid tumors treated with immune checkpoint inhibitors: a systematic review and meta-analysis. J Immunother 2019:43: 1-7.

24. Cortellini A, Buti S, Agostinelli V, et al. A systematic review on the emerging association between the occurrence of immune-related adverse events and clinical outcomes with checkpoint inhibitors in advanced cancer patients. Semin Oncol 2019;46:362-371.

25. Hsiehchen D, Watters MK, Lu R, et al. Variation in the assessment of immune-related adverse event occurrence, grade, and timing in patients receiving immune checkpoint inhibitors. JAMA Netw Open 2019;2: e1911519.

26. Anaya JM. The autoimmune tautology. A summary of evidence. Joint Bone Spine 2017;84:251-253.

27. Khan Z, Hammer C, Guardino E, et al. Mechanisms of immune-related adverse events associated with immune checkpoint blockade: using germline genetics to develop a personalized approach. Genome Med 2019;11:39.

28. Bliddal S, Nielsen $\mathrm{CH}$, Feldt-Rasmussen U. Recent advances in understanding autoimmune thyroid disease: the tallest tree in the forest of polyautoimmunity. F1000Res 2017;6:1776.

29. Eggermont AMM, Kicinski M, Blank CU, et al. Association between immune-related adverse events and recurrence-free survival among patients with stage III melanoma randomized to receive pembrolizumab or placebo: a secondary analysis of a randomized clinical trial. JAMA Onco 2020;6:519-527. 